\title{
A MODULAR ANALOG OF A THEOREM OF R. STEINBERG ON COINVARIANTS OF COMPLEX PSEUDOREFLECTION GROUPS
}

\author{
LARRY SMITH \\ Mathematisches Institut der Universität, Bunsenstraße 3/5, D37073 Göttingen, Federal Republic of Germany \\ e-mail:larry@sunrise.uni-math.gwdg.de
}

(Received 30 May, 2001; accepted 23 July, 2001)

\begin{abstract}
Let $\rho: G \hookrightarrow \operatorname{GL}(n, \mathbb{F})$ be a representation of a finite group over the field $\mathbb{F}, V=\mathbb{F}^{n}$ the corresponding $G$-module, and $\mathbb{F}[V]$ the algebra of polynomial functions on $V$. The action of $G$ on $V$ extends to $\mathbb{F}[V]$, and $\mathbb{F}[V]^{G}$, respectively $\mathbb{F}[V]_{G}$, denotes the ring of invariants, respectively coinvariants. The theorem of Steinberg referred to in the title says that when $\mathbb{F}=\mathbb{C}, \operatorname{dim}_{\mathbb{C}}\left(\operatorname{Tot}\left(\mathbb{C}[V]_{G}\right)\right)=|G|$ if and only if $G$ is a complex reflection group. Here $\operatorname{Tot}\left(\mathbb{F}[V]_{G}\right)$ denotes the direct sum of all the homogeneous components of the graded algebra $\mathbb{E}[V]_{G}$ and $|G|$ is the order of $G$. Chevalley's theorem tells us that the ring of invariants of a complex pseudoreflection representation $G \hookrightarrow \operatorname{GL}(n, \mathbb{C})$ is polynomial algebra, and the theorem of Shephard and Todd yields the converse. Combining these results gives: $\operatorname{dim}_{\mathbb{E}}\left(\operatorname{Tot}\left(\mathbb{C}[V]_{G}\right)=|G|\right.$ if and only if $\mathbb{C}[V]^{G}$ is a polynomial algebra. The purpose of this note is to show that
\end{abstract} the two conditions

(i) $\operatorname{dim}_{\mathbb{F}}\left(\operatorname{Tot}\left(\mathbb{F}[V]_{G}\right)\right)=|G|$,

(ii) $\mathbb{E}[V]^{G}$ is a polynomial algebra

are equivalent regardless of the ground field; in particular in the modular case.

2000 Mathematics Subject Classification. 13A50, 20F55, 20 F56.

Let $\rho: G \hookrightarrow \operatorname{GL}(n, \mathbb{F})$ be a representation of a finite group over the field $\mathbb{F}$, $V=\mathbb{F}^{n}$ the corresponding $G$-module, and $\mathbb{F}[V]$ the algebra of polynomial functions on $V$. The action of $G$ on $V$ extends to $\mathbb{F}[V]$, and we denote by $\mathbb{F}[V]^{G}$, respectively $\mathbb{F}[V]_{G}$, the ring of invariants, respectively coinvariants. As a general reference for invariant theoretic matters we use [2]. The theorem of Steinberg referred to in the title is the equivalence $(a) \Longleftrightarrow\left(e^{\prime}\right)$ in Theorem 1.3 of the amazing paper [3]. It says that when $\mathbb{F}=\mathbb{C}, \operatorname{dim}_{C}\left(\operatorname{Tot}\left(\mathbb{C}[V]_{G}\right)\right)=|G|$ if and only if $G$ is a complex reflection group. Here $\operatorname{Tot}\left(\mathbb{F}[V]_{G}\right)$ denotes the direct sum of all the homogeneous components of the graded algebra $\mathbb{F}[V]_{G}$ and $|G|$ is the order of $G$. Chevalley's theorem tells us that the ring of invariants of a complex pseudoreflection group $G \hookrightarrow \operatorname{GL}(n, \mathbb{C})$ is a polynomial algebra, and the theorem of Shephard and Todd yields the converse, [2, Theorem 7.4.1]. The purpose of this note is to prove the following theorem, which is a characteristic free analog of Steinberg's theorem.

THEOREM. Let $G \stackrel{\rho}{\hookrightarrow} \mathrm{GL}(n, \mathbb{F})$ be a representation of a finite group over the field $\mathbb{F}$. Then the conditions

(i) $\operatorname{dim}_{\mathbb{F}}\left(\operatorname{Tot}\left(\mathbb{F}[V]_{G}\right)\right)=|G|$,

(ii) $\mathbb{F}[V]^{G}$ is a polynomial algebra,

are equivalent. 
Proof. Suppose (i) holds. By definition

$$
\mathbb{F}[V]_{G}=\mathbb{F} \otimes_{\mathbb{F}[V]^{G}} \mathbb{F}[V]
$$

is the module of $\mathbb{F}[V]^{G}$-indecomposable elements of the module $\mathbb{F}[V]$ (see e.g., [2, Section 5.1]). Therefore we can find an epimorphism

$$
\varphi: \mathbb{F}[V]^{G} \otimes \mathbb{F}[V]_{G} \longrightarrow \mathbb{F}[V]
$$

of $\mathbb{F}[V]^{G}$-modules. We claim that $\varphi$ is actually an isomorphism. To see this let $\mathbb{K}=$ $F F\left(\mathbb{F}[V]^{G}\right)$ be the field of fractions of $\mathbb{E}[V]^{G}$ and $\mathbb{L}=F F(\mathbb{F}[V])$ that of $\mathbb{E}[V]$ and note that $\varphi$ induces a map (the classic Cartan-Eilenberg change of rings map)

$$
\Phi: \mathbb{K} \otimes \mathbb{F}[V]_{G}=\mathbb{K} \otimes_{\mathbb{F}[V]^{G}}\left(\mathbb{F}[V]^{G} \otimes \mathbb{F}[V]_{G}\right) \longrightarrow \mathbb{L} \otimes_{\mathbb{F}[V]} \mathbb{F}[V]=\mathbb{L}
$$

of $\mathbb{K}$-vector spaces. We claim $\Phi$ is an epimorphism. For, if $f / h \in \mathbb{L}$, then by multiplying numerator and denominator by $\prod_{1 \neq g \in G} g h$ we may suppose that $h$ is $G$-invariant, so $1 / h \in \mathbb{K}$. Since $\varphi$ is an epimorphism we may write

$$
f=\varphi\left(\sum_{i \in \mathscr{I}} f_{i} \otimes u_{i}\right)
$$

where $\mathscr{I}$ is a finite index set, $f_{i} \in \mathbb{F}[V]^{G}$, and $u_{i} \in \mathbb{F}[V]_{G}$. Then

$$
\frac{f}{h}=\frac{1}{h} \varphi\left(\sum_{i \in \mathscr{I}} f_{i} \otimes u_{i}\right)=\Phi\left(\frac{1}{h} \otimes \varphi\left(\sum_{i \in \mathscr{I}} f_{i} \otimes u_{i}\right)\right),
$$

showing $\Phi$ is an epimorphism.

Since (i) holds, $\mathbb{K} \otimes \mathbb{F}[V]_{G}$ has dimension $|G|$ as a $\mathbb{K}$-vector space, and since $\mathbb{K} \hookrightarrow \mathbb{L}$ is Galois with Galois group $G$ so does $\mathbb{L}$. Hence $\Phi$ is an isomorphism.

The functor $\mathbb{K} \otimes_{\mathbb{F}[V]^{G}}$-is an exact functor, so $\operatorname{ker}(\mathbb{K} \otimes \varphi)=\mathbb{K} \otimes_{\mathbb{F}[V]^{G}} \operatorname{ker}(\varphi)$. Since $\operatorname{ker}(\varphi)$ is $\mathbb{F}[V]^{G}$-torsion free $\operatorname{ker}(\varphi) \neq 0$ implies that $\mathbb{K} \otimes_{\mathbb{F}[V]^{G}} \operatorname{ker}(\varphi) \neq 0$ and this implies $\operatorname{ker}(\Phi) \neq 0$ contrary to what was just shown. Hence $\operatorname{ker}(\varphi)=0$ and $\varphi$ is an isomorphism. Therefore $\mathbb{F}[V]$ is a free $\mathbb{F}[V]^{G}$-module and the result follows from [2, Theorem 6.4.4] or [1].

The implication (ii) $\Rightarrow$ (i) follows from the Degree theorem, [2, Theorem 5.5.3]. Specifically, if $\mathbb{F}[V]^{G}=\mathbb{F}\left[f_{1}, \ldots, f_{n}\right]$ then $f_{1}, \ldots, f_{n} \in \mathbb{F}[V]$ are a system of parameters since $\mathbb{F}[V]^{G} \hookrightarrow \mathbb{F}[V]$ is a finite extension. Since $\mathbb{F}[V]$ is Cohen-Macaulay $f_{1}, \ldots, f_{n} \in$ $\mathbb{F}[V]$ is a regular sequence so $\mathbb{F}[V]$ is a free $\mathbb{F}[V]^{G}$ module. Let $\operatorname{deg}\left(f_{i}\right)=d_{i}$ for $i=$ $1, \ldots, n$. Then the Poincare series of $\mathbb{E}[V]_{G}$ is

$$
P\left(\mathbb{F}[V]_{G}, t\right)=\frac{P(\mathbb{F}[V], t)}{P\left(\mathbb{F}[V]^{G}, t\right)}=\frac{\frac{1}{(1-t)^{n}}}{\frac{1}{\prod_{i=1}^{n}\left(1-t^{d_{i}}\right)}}=\prod_{i=1}^{n}\left(1+t+\cdots+t^{d_{i}-1}\right)
$$

and evaluating $P\left(\mathbb{F}[V]_{G}, t\right)$ at $t=1$ then gives

$$
\operatorname{dim}_{\mathbb{F}}\left(\operatorname{Tot}\left(\mathbb{F}[V]_{G}\right)\right)=d_{1} \cdots d_{n}=|G|
$$

by a corollary to the Degree theorem [2, Corollary 5.5.4]. 
REMARK. The argument in the proof of the theorem that shows that $\Phi$ is an epimorphism is valid in general. It implies the following elementary fact which shows that the condition (i) is an extremal condition, namely a minimum condition.

COROLlary. Let $G \stackrel{\rho}{\hookrightarrow} \operatorname{GL}(n, \mathbb{F})$ be a faithful representation of a finite group over the field $\mathbb{F}$. Then $\operatorname{dim}_{\mathbb{F}}\left(\mathbb{F}[V]_{G}\right) \geq|G|$.

\section{REFERENCES}

1. L. Smith, On the invariant theory of finite pseudoreflection groups, Archiv Math. (Basel) 44 (1985), 225-228.

2. L. Smith, Polynomial invariants of finite groups (A.K. Peters, Ltd., Wellesley, MA, 1995, second printing 1997).

3. R. Steinberg, Differential equations invariant under finite reflection groups, Trans. Amer. Math. Soc. 112 (1964), 392-400. 